\title{
Futur du passé im Französischen der Gegenwart
}

\author{
Von ANGela SCHROTT
}

\section{Vorbemerkungen}

In den letzten Jahren hat sich der sprachliche Ausdruck von Futurität zu einem Schwerpunkt der Tempusforschung entwickelt, die dabei meist die Konkurrenz von futur simple und futur périphrastique in den Mittelpunkt stellt. Die neueren Arbeiten klammern dabei die futur du passé-Formen meist aus und gehen nicht näher auf das durch il allait faire und il ferait versprachlichte Konzept der ,Zukunft in der Vergangenheit ' ein ${ }^{1}$. Diese Abtrennung ist insofern gerechtfertigt, als das futur du passé durch den Transfer in die Vergangenheit einen Verwendungstyp à part darstellt. Dennoch erhebt sich bei einem solchen Transfer natürlich die Frage, inwiefern das semantisch-pragmatische Profil der Formen bei der Verwendung als futur du passé weiter wirkt. Im Zentrum des vorliegenden Beitrags stehen also die semantischen und pragmatischen Werte der futur du passé-Formen und deren mögliche Analogien zu futur simple und futur périphrastique. Die bisher noch weit weniger als die Futura behandelten futur du passé-Formen werden anhand von Beispielen aus Texten des zeitgenössischen Französisch untersucht, in denen Semantik und Pragmatik der Formen durch Kommutationen und Sprecherbefragungen ausgelotet werden ${ }^{2}$. Das besondere Augenmerk gilt dabei Inkongruenzen zwischen den Futur-Formen und den futur du passé-Formen sowie deren Korrelation mit dem veränderten Kontext der, verschobenen“ Futuropposition.

Bevor ich näher auf das futur du passé eingehe, soll als Basis für meine Ausführungen die Futuropposition von futur simple und futur périphrastique in ihren Grundzügen dargestellt werden.

\section{Futur simple vs. futur périphrastique}

In der Forschung herrscht Konsens darüber, daß das futur périphrastique einen zukünftigen Sachverhalt als Folge einer in der Sprechsituation bereits präsenten Vorstufe versprachlicht und damit in Kontinuität zum ego-hic-nunc setzt. Das futur simple dagegen etabliert keine Verbindungen zwischen den versprachlichten

1 Sundell 1991; Helland 1994; Schrott 1997.

2 Mein Dank gilt Claus Morgenstern, der mir von ihm gesammeltes Beispielmaterial überließ und den Aufsatz durch anregende Diskussionen förderte, sowie Claire Chesnais und Malika Séverin, die sich interessiert und ausdauernd für Sprecherbefragungen zur Verfuigung stellten. 
Sachverhalten und der origo und impliziert so eine Zäsur zwischen den futurischen Sachverhalten und der Sprechsituation ${ }^{3}$. Die Bindung an die Gegenwart stellt nun kein rein zeitliches Kriterium dar, sondern kann auch als Inferenzrelation verstanden werden ${ }^{4}$, bei der ein Vorstadium die futurische Handlung bedingt und nach sich zieht - die Realisierung des futurischen Sachverhalts ist also abhängig von der Erfüllung notwendiger Vorbedingungen oder Konditionen 5 . Nach diesem „Prinzip der Konditionierung"6 versprachlicht das futur périphrastique Sachverhalte, bei denen die Bedingungen für die Realisierung bereits erfüllt sind, während Sachverhalte im futur simple von noch unerfüllten Bedingungen abhängen. Futurische Sachverhalte im futur périphrastique, die durch eine Vorstufe bereits in der Sprechsituation präsent sind, haben in der Sprechsituation eine erfüllte, aktuelle conditio und sind damit trotz ihrer Futurität bereits teilaktuell. Das futur simple dagegen hat durch seine Abhängigkeit von unerfüllten Bedingungen eine virtuelle Semantik. Diese Opposition von aktueller und virtueller Konditionierung illustriert das folgende Beispiel:

(1) „Tu m'en veux“, dit Antoine.

Pas de réponse. „Père (va mourir - *mourra)“, reprit Antoine en manière d'excuse. Jacques détourna la tête un instant. „Quand? [...] Quand ... penses-tu partir?“ - „Au plus tôt. Tout est à craindre ...“ (Martin du Gard 1972a, S. 197).

Durch die schwere Krankheit des Vaters ist dessen Ableben bereits aktuell konditioniert, so daß das virtuelle futur simple hier nicht akzeptabel ist. Das futur simple würde lediglich aussagen, daß der Tod des Vaters irgendwann in der $\mathrm{Zu}$ kunft stattfinden wird und damit die Sterblichkeit des Vaters thematisieren.

Im Unterschied zu (1) gibt es nun auch Kontexte, in denen futur simple und futur périphrastique möglich sind, allerdings mit unterschiedlichem semantischpragmatischem Wert:

(2) Elle vient de passer à l'instant, dit-il. Va toujours voir par là, ma mignonne. Tu tournes à gauche, sur le boulevard. En marchant vite, tu (la rattraperas - vas la rattraper), ta maman ... (Troyat 1956, S. 51 ).

Im futur simple erscheint das Einholen der Mutter an eine virtuelle Bedingung geknüpft: das Kind holt sie nur ein, wenn es sich wirklich beeilt. Das futur périphrastique dagegen läßt die Bedingung ,en marchant vite“ als erfüllt erscheinen: das Kind ist hier schon auf dem Weg und die Tatsache des Einholens erscheint gesichert.

Die virtuelle bzw. teilaktuelle Konditionierung bedingt ferner, daß Sachverhalte im futur simple und futur périphrastique auf unterschiedliche Weise in der

3 Damourette/Pichon 1936, 164, 169, 276-284; Imbs 1960, 55; Togeby 1982, 396-399; Fleischman 1982, 96 ff.; Sundell 1991, 8, 21; Helland 1994, 252 f., 263; Franckel 1984, $66 \mathrm{f}$, , 68; Schrott 1997, 26-40.

4 Fleischman 1982, 95; Sundell 1991, 88.

5 Flydal 1943, 48 f., 51 f., 100; Diese Unterscheidung Flydals liegt auch den Ausfuihrungen von Morgenstern 1988 zugrunde. Vgl. auch Sten 1952, $233 \mathrm{f}$.

$6 \mathrm{Vgl}$. dazu ausführlicher Schrott 1997, 26-40, bes. $30-32$. 
Zukunft lokalisiert werden ${ }^{7}$. Da beim futur périphrastique die Realisierung durch die Vorstufe gleichsam schon begonnen hat, ist ein bestimmter Zeitpunkt der Durchführung präsupponiert, der futurische Sachverhalt ist orientiert. Das futur simple dagegen trifft lediglich die Existenzaussage, daß der Sachverhalt, irgendwann' in der Zukunft realisiert werden wird, und enthält keine zeitliche Orientiertheit:

(3) „En ce moment, dit Flavie, je crois que j'aurais besoin de quelque chose. Je ne me sens pas très bien. Cette histoire m'a toute retournée ...“ „Attendez, madame, je (vais vous donner - ?*vous donnerai) un cordial" (Chevallier 1968, S. 33 f.).

Da Flavie akut an Unwohlsein leidet, muß das Verabreichen des „cordial“ eine orientierte Handlung darstellen, die in einem bestimmten Zeitpunkt erfolgt. Das futur simple dagegen würde implizieren, daß der Arzt der Frau das Medikament irgendwann später geben wird, was in diesem Kontext nicht gemeint ist. Das futur périphrastique beinhaltet außerdem, daß der Arzt Flavie das Medikament unverzüglich geben wird. Da teilaktuelle futurische Sachverhalte nicht mehr von der Erfüllung anderer Bedingungen abhängen, werden sie in Kontiguität zur Sprechsituation realisiert, also ohne daß sich noch andere intermittierende Sachverhalte dazwischenschieben ${ }^{8}$.

Auch die Virtualität des futur simple schlägt sich in einer typischen Ereignisstruktur nieder. Da Sachverhalte im virtuellen futur simple von unerfüllten Bedingungen abhängen, versprachlicht das futur simple häufig Sachverhalte, die erst nachzeitig zu einem anderen Sachverhalt eintreten können und damit in einer Struktur der Ulteriorität ${ }^{9}$ zur origo stehen. Dies ist im nächsten Beispiel der Fall, wo ein auf einer Leiter stehender Mann mit der Versicherung beruhigt wird, man werde ihn im Falle eines Sturzes schon wieder aufsammeln ${ }^{10}$ :

(4) Monsieur n'a qu'à se laisser tomber. On (le ramassera - *va le ramasser) après.

Der im futur simple versprachlichte Sachverhalt „On le ramassera“ ist nicht nur nachzeitig zum Sturz des Mannes, der mögliche Sturz stellt auch die noch zu erfüllende virtuelle Bedingung dar.

Die virtuelle und aktuelle Konditionierung der Futura manifestiert sich auch in einer aspektuellen Markierung ${ }^{11}$, die ausgehend von Comries Modell des imperfektiven Aspekts erfaßt werden soll ${ }^{12}$. Der imperfektive Aspekt fokussiert die interne Struktur eines Sachverhaltes, ohne die Außengrenzen einzubeziehen; kon-

7 Analoge Differenzierungen finden sich bei Flydal 1943, 31; Vet 1980, 83, 98; Franckel 1984, 67.

8 Vgl. dazu Franckel 1984, 66 ff.; Schrott 1997, 42-44.

9 Vgl. dazu näher Schrott 1997, $42 \mathrm{f}$.

10 Beispiel Wainstein 1949, 141.

11 Zum Begriff des Aspekts vgl. etwa Comrie 1976, 5; Coseriu 1976, 115; Vet 1980, 46-54, 77-80; Fleischman 1982, 11.

12 Comrie 1976, 4, 21 ff., 24 f. Vgl. zur aspektuellen Markierung der Futura auch Schrott 1997, 101-117. 
trastiv dazu blendet der perfektive Aspekt die interne Struktur aus und rückt die Außengrenzen, Anfang und Ende, ins Zentrum. Beim imperfektiv markierten französischen imparfait bewirkt dies, daß ein Sachverhalt als, schon und noch im Vollzug' gesehen wird, wogegen perfektive Formen wie etwa das französische passé simple als anfangs- und/oder endterminiert erscheinen. Dem futur périphrastique kann nun ein prospektiver Aspekt zugeordnet werden ${ }^{13}$, der durch eine Hervorhebung einer Vorstufe oder Anfangsphase charakterisiert ist, aus der sich dann ein Sachverhalt entwickelt. Diese akzentuierte Anfangsphase entspricht nun in meinem Modell der aktuellen conditio, die ja ebenfalls eine Vorstufe darstellt, so daß der prospektive Aspekt als Effekt der aktuellen Konditionierung aufgefaßt werden kann. Dem futur simple dagegen fehlt die Basis für diese aspektuelle Markierung, so daß es hinsichtlich des prospektiven Aspektes unmarkiert ist. Da das futur périphrastique durch die Fokussierung der Anfangsphase dem ausgedrückten Sachverhalt eine interne Struktur aufprägt, stellt der prospektive Aspekt einen Typ von Imperfektivität dar ${ }^{14}$. Im Unterschied zum futur simple enthält das futur périphrastique also neben seiner temporal-deiktischen auch eine aspektuelle Komponente. Diese beiden Werte sind in allen Verwendungen des futur périphrastique kopräsent, doch kann die aspektuelle Komponente in bestimmten Kontexten besonders akzentuiert sein ${ }^{15}$ :

(5) [...] Il a passé tout à côté de moi, avec sa lourde serviette qui lui remontait l'épaule, son canotier, sa jaquette noire [...] Il aura toujours l'air d'un professeur qui (va faire $-{ }^{*}$ fera) sa classe [...] (Martin du Gard 1972b, S. 105).

Beschrieben wird der lehrerhafte Habitus des Politikers Jaurès, der den Eindruck vermittelt, im nächsten Augenblick mit dem Dozieren zu beginnen - dieser prospektive Wert kann nur vom futur périphrastique geleistet werden.

\section{Die Funktionsweise des futur du passé}

Bevor die Opposition von futur simple du passé und futur périphrastique $d u$ passé und mögliche Modifizierungen gegenüber der Futuropposition behandelt werden, sind einige Prämissen der Funktionsweise des futur du passé zu klären ${ }^{16}$.

13 Comrie 1976, 64, Fleischman 1982, 18 f., 95-98, bes. 96 f.; vgl. auch Coseriu 1976, $103 \mathrm{f}$., bei dem der prospektive Aspekt der imminentiellen, ingressiven Phase entspricht. Vgl. ebenfalls Schrott 1997, 105-108.

14 Die Einordnung der Prospektivität als imperfektiver Aspekt wird auch durch das Faktum untermauert, daß das futur périphrastique der Form je vais faire nur noch als futur $d u$ passé der Form j'allais faire existiert. In beiden Fällen ist das ins présent bzw. imparfait gesetzte Auxiliar damit imperfektiv markiert. Das futur simple dagegen könnte im Sinne Comries als perfektiv eingestuft werden, da es keine interne Strukturierung leistet. Vgl. dazu auch Schrott 1997, 110.

15 Vgl. Comrie 1976, 64 und Vet 1994, 54, 59. Aufgrund dieser graduellen Akzentuierung sollten Tempus und Aspekt beim futur périphrastique nicht als getrennte Werte betrachtet werden, wie dies Gougenheim 1929, $106 \mathrm{ff}$. und Flydal 1943, 18 ff. tun. Vgl. zum Verhältnis von Tempus und Aspekt auch Schrott 1997, 115-117. Die Kopräsenz von 
Da futur simple und futur périphrastique in funktioneller Opposition zu anderen Verbalparadigmen stehen und Teil des Tempussystems sind, können sie nur sehr beschränkt andere Werte des Systems annehmen ${ }^{17}$. So treten die Futura im Verbalsystem nur noch in einer, Verschiebung in die Vergangenheit" in der Funktion futur du passé auf. Wie das futur simple so fungiert auch das futur périphrastique nur noch als futur du passé, wobei das konjugierte Auxiliar als Ausdruck der temporalen Markierung vom présent ins imparfait gesetzt wird ${ }^{18}$.

Wie die Futura so haben auch die futur du passé-Formen den Grundwert, Sachverhalte zu versprachlichen, die zu einer Referenzsituation nachzeitig sind. Bei den Futura ist diese Relation der Nachzeitigkeit auf das ego-hic-nunc als Zentrum der aktuellen Welt bezogen, in der auch die Sprecherinstanz lokalisiert ist. Die futur du passé-Formen dagegen sind auf eine Referenzsituation bezogen, die nicht mit der Sprecher-origo identisch ist, sondern vorzeitig zum ego-hic$n u n c$ in der Vergangenheit situiert ist ${ }^{19}$. Diese Referenzsituation wird durch den Kontext etabliert und in Relation zum ego-hic-nunc in der Vergangenheit verortet - sie bildet das Zentrum einer inaktuellen, erzählten Welt. Im Unterschied zu futur simple und futur périphrastique sind die futur du passé-Formen damit nur indirekt über ihre Referenzsituation in der Vergangenheit mit der origo verbunden ${ }^{20}$. Dabei ist die zeitliche Relation zwischen dem vom futur du passé versprachlichten nachzeitigen Sachverhalt und der origo nicht festgelegt. Dieser ,blinde Fleck' der Tempussemantik wird erst durch den jeweiligen Kontext determiniert ${ }^{21}$. Für das Funktionieren der futur du passé-Formen entscheidend ist nun, daß dem Konzept der Nachzeitigkeit zu einer Referenzsituation in der Vergangenheit eine Perspektive inhärent ist, nämlich der Blick von dieser Referenzsituation in die ,Zukunft' der nachzeitigen Sachverhalte ${ }^{22}$. Durch diese Perspektive eines Denkens an die Zukunft, das der Vergangenheit angehört ${ }^{23}$, beinhaltet das futur du passé einen Standpunkt in der Vergangenheit, von dem aus Zukünftiges bzw.

aspektueller und temporal-deiktischer Komponente ist auch erwartbar, wenn man einbezieht, daß sich das futur périphrastique diachronisch aus einem Aspekt entwickelt hat, vgl. hierzu etwa Fleischman 1982, 85, 89.

16 Zur historischen Entwicklung von futur simple du passé und futur périphrastique $d u$ passé vgl. Wilmet 1970, 399-416, 417-421.

17 Spang-Hanssen 1983, 15 f.; Flydal 1943, 61; Togeby 1982, 392.

18 Vgl. Togeby $1982,393$.

$19 \mathrm{Zu}$ dieser Verschiebung des Zentrums als einer Unterscheidung von Aktualität der Sprechsituation und Inaktualität vgl. vor allem Vet 1980, $31 \mathrm{f}$. (monde actuel vs. monde non actuel); Martin 1971, 142; Coseriu 1976, $92 \mathrm{f}$. (aktuelle vs. inaktuelle Ebene); Weinrich 1982, 161 (besprochene vs. erzählte Welt). Vgl. ferner Reichenbach 1947, 293 f.; Comrie 1985, 114, 128; Roncador 1988, 181 f., 171.

20 Comrie 1985, 36, 56, 65; 123, 128.

${ }^{21}$ Comrie 1985, 128; vgl. auch Tobler ${ }^{2} 1906,143-144,146$.

22 Vgl. dazu auch Tobler ${ }^{2}$ 1906, 141; Gougenheim 1929, 108-109; Flydal 1943, 65, 67; N. M. Steinberg 1972, 177-178; Vet 1980, 32.

${ }^{23}$ Tobler ${ }^{2} 1906,141$ nennt diese Sicht „ein der Vergangenheit angehörendes Denken an Zukünftiges“. 
Nachzeitiges anvisiert wird ${ }^{24}$. Eine solche Perspektive setzt nun voraus, daß es auch einen Träger dieser Perspektive gibt, der in der Referenzsituation präsent ist. Das von den futur du passé-Formen versprachlichte Konzept der Nachzeitigkeit impliziert also einen Perspektivträger dieser Nachzeitigkeit, so daß das futur $d u$ passé als perspektivgebundene Form bezeichnet werden kann. Auf diesen temporalen Wert von il allait faire und il ferait werde ich mich in dieser Abhandlung konzentrieren und modale Verwendungstypen des futur périphrastique du passé und des ,conditionnel" futur simple du passé ausklammern ${ }^{25}$.

Der temporale Grundwert der futur du passé-Formen soll anhand des folgenden Beispiels illustriert werden, in dem ein Ich-Erzähler Erlebnisse seiner Kindheit erzählt:

(6) Je déclarai donc que j'allais partir tout seul pour le Taoumé, afin d'observer le passage des oiseaux, et de choisir les emplacements de nos pièges, et je chargeai ma mère de dire à Lili, lorsqu'il viendrait vers quatre heures, que je l'attendrais à la cabane des charbonniers, au pied de l'éperon du Taoumé (Pagnol 1988c, S. 163).

Durch den im verbum dicendi ,je déclarai“ versprachlichten Äußerungsakt wird kontextuell eine Referenzsituation in der Vergangenheit konstituiert ${ }^{26}$, zu der dann die durch futur périphrastique du passé und futur simple du passé angekündigten Pläne des Jungen nachzeitig sind. Für das erzählte Ich, den in der Referenzsituation präsenten Protagonisten also, sind diese Handlungen zukünftig - die Rolle des Perspektivträgers wird also hier vom Protagonisten, dessen Rede referiert wird, erfüllt.

Als nächstes gilt es zu prüfen, wie sich die Opposition der futur du passéFormen gestaltet und inwiefern die für die Opposition von futur simple und futur périphrastique erarbeiteten Charakteristika auch für die futur du passé-Formen Gültigkeit haben. Dabei werde ich mit Kontexten beginnen, in denen wie im besprochenen Beispiel (6) die Funktion des Perspektivträgers durch einen Protagonisten erfüllt wird.

\section{Die Perspektive des Protagonisten}

Die Untersuchung der futur du passé-Formen soll zunächst auf Strukturen begrenzt werden, in denen die Funktion des Perspektivträgers mit einem Prot-

24 Das Konzept einer solchen Perspektive findet sich in Ansätzen bei Damourette/Pichon 1936, 429; Martin 1971, 123; G. Steinberg 1971, 245; Weinrich 1982, 203.

$25 \mathrm{Zu}$ den modalen Werten des „conditionnel futur du passé" vgl. Damourette/Pichon 1936, 431-449; Sten 1952, 79-94; Imbs 1960, 70-89; Martin 1971, 126-131; Togeby 1982, 388-390; Dendale 1993, bes. 166-168, 173 f. Zum Zusammenhang zwischen temporalen und modalen Werten vgl. Tobler ${ }^{2} 1906,157 \mathrm{f}$; Damourette/Pichon 1936, 408-409; Wilmet 1970, 410 f., 415; Sten 1952, 83 f. Auf modale Werte des futur périphrastique du passé wird dagegen nur selten eingegangen, so bei Gougenheim 1929, 109 und Flydal 1943, $64 \mathrm{f}$.

26 Martin 1971, 123. 
agonisten besetzt ist, der sich zu für ihn zukünftigen Sachverhalten äußert, wobei eine solche Protagonistenrede in Worten oder Gedanken realisiert sein kann. Entscheidend ist die Bindung der Rede an einen Protagonisten, der sich in der vergangenen Referenzsituation zu nachzeitigen Geschehnissen äußert und dessen Rede indirekt wiedergegeben wird. In diesen Strukturen ist der Perspektivträger also ein Protagonist als „eine bestimmte Person der zurückliegenden Zeit" 27 .

Solche Fälle der Protagonistenrede werden in der Forschung als die Kontexte beschrieben, in denen beide futur du passé-Formen struktural verwendbar sind und eine zu den Futura analoge Opposition bilden ${ }^{28}$. Die Beschränkung dieser funktionierenden Opposition auf Protagonistenrede wird mit einer Restriktion des futur simple du passé begründet. Die Forschung attestiert dem futur simple du passé nämlich, daß es ausschließlich in Protagonistenrede verwendet wird. Damit erscheint auch die Opposition der futur du passé-Formen auf Strukturen der indirekt wiedergegebenen Protagonistenrede beschränkt, wie sie beim style indirect und beim style indirect libre vorliegen ${ }^{29}$. Bevor ich auf diese in der Forschung postulierte Restriktion, deren Gültigkeit noch zu hinterfragen ist, zurückkomme, soll die Ausgestaltung der futur du passé-Opposition zunächst in Strukturen indirekter Rede untersucht werden.

\subsection{Protagonistenrede: der Typ Je déclarai que $p$}

Die Analyse von Kontexten der Protagonistenrede beginnt mit Beispielen syntaktisch untergeordneter indirekter Rede, wie sie ja auch im bereits besprochenen Beispiel (6) vorlag. Das redeeinleitende Verb in einem Tempus der Vergangenheit liefert einen acte d'énonciation, der dann die vergangene Referenzsituation konstituiert, in der der Protagonist als Perspektivträger lokalisiert ist. Die Äußerung des Protagonisten wird dann in einer mit que eingeleiteten phrase complétive wiedergegeben ${ }^{30}$. Die durch das Vergangenheitstempus etablierte Referenzsituation in der Vergangenheit bedingt nun einen Tempustransfer. Dabei werden die Tempora der zugrundeliegenden origo-bezogenen direkten Rede an das neue Zentrum in der inaktuellen Welt adaptiert. Nachzeitige Sachverhalte, die in der direkten Rede durch futur simple und futur périphrastique ausgedrückt werden, werden dann aufgrund des Transfers der Referenzsituation durch die futur du passé-Formen versprachlicht. Der Sprecher bzw. Erzähler transponiert die Tempora also und macht sie zu einem Teil der erzählten Welt ${ }^{31}$. Der referierte acte d'énoncia-

27 Tobler ${ }^{2} 1906,141$.

28 Gougenheim 1929, 108 f.; Flydal 1943, 67; Nilsson-Ehle 1944, 55-57, 62-64; Sten 1952, 72, 74; Togeby 1982, 294 ff., 383 ff., 402 f. Zahlreiche Beispiele für futur simple du passé und futur périphrastique du passé in Protagonistenrede liefern Damourette/ Pichon 1936, 322-326, 407-430.

29 Zur Charakterisierung indirekter Rede allgemein vgl. Pollak 1960, 178; Roncador 1988, 5-7, 53-66, 102-107; Gather 1994, 207-240.

$30 \mathrm{Vgl}$. Togeby $1982,383,402$.

31 G. Steinberg 1971, 245. Zur Tempustransposition und den konkurrierenden Erklärungsmodellen (deiktische Verschiebung vs. syntaktischer Regelapparat) vgl. vor allem Comrie 1985, 108-114; vgl. ferner Bally 1914, 417 f.; Pollak 1960, 183 f.; G. Steinberg 1971, 243-255; Gather 1994, 217 f. 
tion des Protagonisten ist damit in eine weitere situation d'énonciation eingebettet, in der ein Sprecher bzw. Erzähler über diesen Äußerungsakt in der Vergangenheit berichtet.

Im folgenden beschränke ich die Untersuchung der futur du passé-Opposition also auf Fälle, in denen die Protagonistenperspektive aus der Vergangenheit in die Zukunft durch die syntaktisch untergeordnete indirekte Rede modelliert ist. Anhand von Beispielanalysen läßt sich belegen, daß die bei den Futura distinktive Opposition von virtueller und aktueller Konditionierung auch beim futur du passé ausschlaggebend ist:

(7) A cette annonce, Paul fut saisi d'un accès de rire en trois quintes si violentes qu'elles dévièrent une bouchée de sardines à la tomate, et je crus qu'il (allait périr - *périrait) sous nos yeux; mais quelques tapes dans le dos lui permirent de retrouver sa respiration (Ebda. S. 88).

Aus der Sicht des Sprechers Marcel ist zu befürchten, daß Paul sich vor Lachen so heftig verschluckt hat, daß man um sein Leben fürchten muß. Das futur simple $d u$ passé wäre hier rein virtuell und würde versprachlichen, daß Paul als sterblicher Mensch irgendwann später einmal unter den Augen der Familie verscheiden wird.

War im obigen Beispiel nur eine Form möglich, so sind im nächsten Textausschnitt beide Formen akzeptabel, mit der bereits bei futur simple und futur périphrastique konstatierten Bedeutungsdifferenzierung:

(8) Dans l'après-midi même, quand j'avais eu la sottise de dire que (j'allais mentir - je mentirais) à Paul, parce que c'était pour son bien, l'oncle Jules avait saisi la balle au bond (Pagnol 1988b, S. 164).

Das futur périphrastique du passé des Originals versprachlicht, daß die Handlung des Lügens bereits als Intention Teilaktualität hat und daß Marcel seinen Bruder in einer bestimmten Angelegenheit belügen wird. Im weiteren Kontext des Romans geht es nämlich darum, den kleinen Bruder über den Termin einer geplanten Jagd zu täuschen. Das futur simple du passé dagegen würde versprachlichen, daß Marcel irgendwann später einmal lügen wird.

Analog zu futur simple und futur périphrastique sind auch die futur du passéFormen durch die Ereignisstrukturen der Kontiguität und Ulteriorität charakterisiert. Im folgenden Beispiel hat der Sprecher einen Jungen, der ihn beleidigte, zum Kampf gefordert:

(9) J'avais compté sur ce préambule, prononcé sur un ton agressif, pour intimider l'adversaire, et j'espérais vaguement qu'il (allait faire - *ferait) de plates excuses (Ebda. S. 273).

Vor einer Schlägerei mit einem gefährlichen Gegner hofft der Junge auf eine Entschuldigung - diese soll unverzüglich, in Kontiguität zur Referenzsituation erfolgen, da der mittlerweile etwas ängstliche Protagonist auf eine gütliche Einigung zählt. Im Falle des futur simple du passé dagegen würde die Entschuldigung nach dem Kampf geliefert werden.

Das Prinzip der Konditionierung bedingt auch bei den futur du passé-Formen, daß das futur périphrastique du passé bei Sukzessionen häufig einen Sachverhalt versprachlicht, der einer Situation im futur simple du passé vorausgeht: 
(10) [...] ils sentaient bien, évidemment, que quelque chose était changé. Beaucoup cependant espéraient toujours que l'épidémie allait s'arrêter et qu'ils seraient épargnés avec leurs familles. En conséquence, ils ne se sentaient encore obligés à rien (Camus 1972, S. 90).

Die Sequenz von futur périphrastique du passé und futur simple du passé versprachlicht, daß das Ende der Epidemie die Voraussetzung dafür ist, daß die Menschen und ihre Familien verschont werden.

Analog zu den Futura manifestiert sich auch beim futur du passé die Konditionierung in der bereits erläuterten Orientiertheit der Sachverhalte. Die aktuelle Konditioniertheit impliziert auch beim futur périphrastique du passé, daß die Sachverhalte in einer bestimmten Situation nachzeitig zur Referenzsituation stattfinden werden:

(11) Raymond lui a expliqué alors que le chien avait pu s'égarer et qu'il (allait revenir - ?reviendrait) (Camus 1968, S. 60).

Während das futur périphrastique du passé die Rückkehr des entlaufenen Hundes als orientierten Sachverhalt versprachlicht, der sich in einem nachzeitigen Zeitpunkt realisieren wird, sagt das futur simple du passé lediglich aus, daß der Hund irgendwann zu seinem Herrn zurückfinden wird. Das orientierte futur périphrastique du passé wird in diesem Fall vorgezogen, da Raymond den Gesprächspartner ja trösten will.

Das besprochene Kriterium der Orientiertheit enthält nun ein pragmatisches Potential, das bei Futura und futur du passé analog funktioniert ${ }^{32}$. Im Beispiel (12) will ein Reisender den Hausherrn sprechen und in (13) wird der Kommissar Maigret vom Untersuchungsrichter erwartet:

- Il n'est pas là, le patron? dit-il en s'asseyant.

- Il (va revenir - *reviendra), dit la femme, mise en confiance. Il est allé chercher un fagot (Alain-Fournier 1971, S. 50 f.).

- Le juge d'instruction a téléphoné deux fois.

- A quelle heure?

- La première fois, un peu après neuf heures, la seconde il y a quelques minutes. La seconde fois, j'ai répondu que vous (alliez descendre - ?*descendriez) (nach Simenon 1989, S. 605).

In beiden Beispielen versprachlicht das futur périphrastique (12) bzw. das futur périphrastique du passé (13), daß das von der Referenzsituation aus nachzeitige Kommen einer Person aktuell konditioniert und orientiert ist. Durch diese Semantik der Orientiertheit erscheint die Rückkehr des patron (12) als ,nah' und dem Ankömmling wird signalisiert, daß das Warten sich lohnt. In (13) wird dieses Signal in der indirekten Rede referiert: der Sprecher erklärt gegenüber Maigret, daß er dessen Kommen als teilaktuell und orientiert versprachlicht hat und den Untersuchungsrichter so zum Warten ermutigt hat. Daraus ergibt sich dann indirekt für Maigret die Verpflichtung, in absehbarer Zeit den Richter aufzusuchen.

32 Vgl. Schrott 1997, 63-64, $201-203$. 
Futur simple und futur simple du passé dagegen beinhalten eine nicht-orientierte Rückkehr und wären in den Beispielen (12) und (13) nur dann adäquat, wenn der Gesprächspartner auf unbestimmte Zeit vertröstet werden soll.

Die beim futur périphrastique konstatierte prospektive Markierung ist auch beim futur périphrastique du passé feststellbar und steht auch hier in Opposition zu einem aspektuell nicht markierten futur simple du passé. Der aspektuelle Wert modelliert sich besonders deutlich heraus, wenn durch den Kontext die Anfangsphase zusätzlich fokussiert ist:

(14) Jenny, qui était derrière Jacques, vit ses épaules tressaillir. Elle crut qu'il (allait intervenir - *interviendrait) dans le débat. Mais il se retourna vers elle, sans rien dire (Martin du Gard 1972b, S. 301).

In (14) ist eine Vorstufe des Intervenierens durch das Zucken der Schultern bereits gegeben - akzentuiert ist also der Moment, in dem Jacques drauf und dran ist, in das Geschehen einzugreifen, das Vorstadium aber dann doch nicht in die Phase der eigentlichen Realisierung eintritt. Auf dieses häufig mit der Aspektfokussierung gekoppelte Handlungsmuster werde ich noch ausführlicher zurückkommen.

Die durchgeführten Beispielanalysen belegen, daß in Strukturen des Typs Je déclairai que $p$ die Formen futur simple du passé und futur périphrastique du passé analog zu den Futura funktionieren und durch das Prinzip der Konditionierung und seine Ausprägungen in Ereignisstruktur, Orientiertheit und Aspektmarkierung charakterisiert sind. Es bleibt zu prüfen, ob diese Analogie zu den Futura auch in der zweiten Struktur der Protagonistenrede besteht, dem style indirect libre.

\subsection{Protagonistenrede im style indirect libre}

Der style indirect libre 33 ist eine frei angeschlossene Form der indirekten Rede, die durch Kontextelemente als Protagonistenrede ausgewiesen ist. So sind im style indirect libre referierte Worte oder Gedanken des Protagonisten häufig auf ein verbum dicendi oder cogitandi bezogen, allerdings ohne eine syntaktische Unterordnung durch que. Dieser formale Typ indirekter Redewiedergabe kann dann in eine Erzähltechnik der perspektivischen Differenzierung übergehen, die „erlebte Rede“, bei der in die Erzählerrede kontrastiv die Rede eines Protagonisten eingeführt wird ${ }^{34}$.

Beim style indirect libre liegt also auch eine Protagonistenrede vor, die durch den ihr impliziten acte d'énonciation eine Referenzsituation in der Vergangenheit

33 Zum Terminus vgl. G. Steinberg 1971, 111-113. Zur syntaktischen und stilistischen Charakterisierung des style indirect libre vgl. Bally 1912, 552-555; Bally 1914, 410414; Lerch 1914, 461-462, 472 f.; Pollak 1960, 181-194; Stempel 1972, 325; Togeby 1982, 383, 386; Roncador 1988, 128-151; Vetters 1994, 179-221; Gather 1994, $231-$ 240.

34 Zur Abgrenzung von style indirect libre als Form der indirekten Rede und ,erlebter Rede“ als Erzähltechnik vgl. Stempel 1972, 311, 315 f., 318, 321, 329; Lebsanft 1981, 71. 
konstituiert. In dieser Referenzsituation ist als Perspektivträger der Protagonist lokalisiert, dessen Denken und Reden nachzeitigen Situationen gilt. Da damit die perspektivische Gebundenheit an einen Protagonisten gegeben ist, sollten die Voraussetzungen für eine funktionierende futur du passé-Opposition erfüllt sein. Diese Annahme wird durch die folgenden Beispielanalysen bestätigt:

(15) Mais, aussitôt, le sentiment d'avoir commis une irrémédiable faute lui sauta aux yeux avec tant d'évidence qu'il en eut la respiration coupée. Quelques mots de trop, tout était compromis: Jacques (allait lui échapper - ?*lui échapperait) définitivement ... (Martin du Gard 1972a, S. 219).

Da durch die bereits gefallenen unbedachten Worte der Verlust seines Bruders Jacques für den Protagonisten Antoine bereits aktuell konditioniert ist, kann das virtuelle futur simple du passé hier nicht verwendet werden. Im nächsten Beispiel dagegen erfordert der Kontext die virtuelle Semantik des futur simple du passé:

(16) Sa pensée, depuis son réveil, ne quittait pas Crouy. En filant sans délai, il pouvait prendre l'express de 14 heures. Il (arriverait - *?allait arriver) à Crouy au jour, mais après la cérémonie, longtemps même après le départ du train de retour: il était donc absolument assuré de ne plus y rencontrer personne. Il (irait - *?allait aller) droit au cimetière et (reviendrait - *?allait revenir) aussitôt (Ebda. S. 392 f.).

Das futur simple du passé des Originals versprachlicht die Planungen als eher hypothetische Überlegungen (,il pouvait“), die an die Bedingung ,en filant sans délai“ geknüpft sind - die nachzeitigen Handlungen erscheinen so als rein virtuelle Überlegungen. Aufgrund der kontextuell geforderten virtuellen und hypothetischen Natur wird das futur périphrastique du passé nicht akzeptiert.

Auch die Ereignisstrukturen der Kontiguität und Ulteriorität prägen sich im style indirect libre je nach Art der Konditionierung aus:

(17) L'auto ralentit et s'arrêta devant une maison à porte basse. Le trottoir était mal éclairé et désert. Jacques crut qu'elle allait lui demander de monter chez elle. (Que ferait-il - Qu'allait-il faire)? (Ebda. S. 406).

Der style indirect libre folgt hier auf eine durch das verbum cogitandi (,Jacques crut") eingeleitete complétive und setzt diese Protagonistenrede unter Wegfall der syntaktischen Unterordnung fort. Die Opposition der futur du passé-Formen entspricht dem Prinzip der Konditionierung. Das futur simple du passé des Originals versprachlicht eine Reaktion auf das erwartete Angebot der Frau, das hier als virtuelle conditio funktioniert - es liegt also eine Ereignisstruktur der Ulteriorität vor. Beim ebenfalls akzeptablen futur périphrastique du passé dagegen würde sich der Protagonist fragen, was er jetzt, in Kontiguität zur Sprechsituation, tun soll, bevor die Frage gestellt wird, also etwa ob er sich rasch verabschieden soll, bevor die Lage schwierig wird.

Die aktuelle und virtuelle Konditionierung manifestiert sich neben der aufgezeigten Ereignisstruktur auch im style indirect libre im Kriterium der Orientiertheit:

(18) C'est à ces moments-là seulement qu'elle le sentait en sa possession. „Mon Tony ..." Il (allait venir - viendrait). Elle en avait la certitude. Il (viendrait - allait venir) ce soir. Elle ne se trompait pas (Martin du Gard 1972b, S. 265). 
In diesem Beispiel wird der gleiche Sachverhalt, die Rückkehr Tonys, einmal im futur périphrastique du passé und dann im futur simple du passé in Kombination mit der temporalen Angabe ce soir versprachlicht. Durch das futur périphrastique du passé wird die Rückkehr als orientiert dargestellt: Tony wird nicht irgendwann zurückkehren, sondern zu einem bestimmten Zeitpunkt. Diese Orientiertheit wird dann in der Wiederholung durch ,il viendrait ce soir" ausgedrückt. Die NichtOrientiertheit des futur simple du passé wird durch die Angabe ce soir kompensiert, die ja eine zeitliche Verankerung nachzeitig zur Referenzsituation leistet. Die Differenz der futur du passé-Formen erscheint in Kombination mit ce soir daher abgeschwächter, wird doch durch den Kontext die Orientiertheit geliefert. $\mathrm{Zu}$ unterstreichen ist, daß auch hier aktuelle bzw. virtuelle Konditionierung unterschiedliche Nuancen bedingen. Eine aktuell konditionierte Rückkehr erscheint aus Protagonistensicht gewisser als eine virtuell konditionierte, so daß der Wechsel der Formen hier auch das Schwanken der Protagonistin illustriert.

Die Beispielanalysen bestätigen das gängige Postulat, daß in style indirect und style indirect libre die futur du passé-Opposition analog zu den Futura funktioniert. Das Prinzip der Konditionierung und seine Ausprägungen in Ereignisstruktur, (Nicht-)Orientiertheit und Aspektmarkierung manifestieren sich in diesen Strukturen der Protagonistenrede ganz analog zu futur simple und futur périphrastique. Diese Kongruenz läßt die futur du passé-Formen als Resultat eines Transfers der Futura in die Vergangenheit erscheinen, bei dem das semantisch-pragmatische Profil der ,primären', auf die origo zentrierten Futura unangetastet bleibt.

$\mathrm{Zu}$ klären bleibt nun, wie sich die futur du passé-Opposition in Kontexten gestaltet, in denen ein Sprecher, der nicht mit einem Protagonisten identisch ist, von einer Referenzsituation in der Vergangenheit aus in die Zukunft blickt.

\section{Die Perspektive des Erzählers}

Wurden bisher Strukturen der Protagonistenrede untersucht, so sollen jetzt Äußerungen im Zentrum der Überlegungen stehen, die nicht auf die énonciation eines Protagonisten zurückführbar sind. Dies ist in unabhängigen Hauptsätzen der Fall, die als style direct ${ }^{35}$ auf einen ,Sprecher' ${ }^{6}$ urückgehen, der kein Protagonist ist und damit im weitesten Sinne eine Erzählerfigur darstellt ${ }^{36}$ :

(19) En 1785, Beaumarchais allait franchir une des dernières étapes de sa vie. Il était dans sa cinquante-troisième année.

Die zur Referenzsituation nachzeitigen Ereignisse werden hier aus der Sicht des Erzählers berichtet und gehen nicht auf einen Protagonisten zurück. Geht man von der Prämisse der Forschung aus, daß das futur simple du passé auf Protagonistenrede begrenzt ist, dann folgt daraus, daß in Kontexten des Typs (19) allein das futur périphrastique du passé verwendet wird, mit der Konsequenz, daß die

35 Togeby $1982,382 \mathrm{f}$, $386 \mathrm{f}$.

36 Beispiel Togeby 1982, 403. Der Begriff ,Erzähler ' meint allgemein den ,Sprecher` des Textes. 
futur du passé-Opposition kollabiert ${ }^{37}$. Diese bisher von der Forschung postulierte beschränkte Verwendung wird durch eine Ausnahme zur Regel ergänzt. Das futur simple du passé findet sich nämlich auch in unabhängigen Hauptsätzen, die nicht als style indirect libre einzustufen sind, sondern vielmehr Erzählerrede darstellen. Dieser Verwendungstyp wird als „,conditionnel historique“ bezeichnet ${ }^{38}$. Die als jüngere Entwicklung eingestufte Verwendung wird als textsortengebundene Ausnahme betrachtet, die ihren Ort in historischen Texten hat ${ }^{39}$. In dieser Textsorte wird das Vorkommen des futur simple du passé dann als „variation stylistique“ zur devoir-Periphrase gedeutet ${ }^{40}$ und damit durch ein Motiv erklärt, das nicht im Wert der Tempusform selbst gründet.

Die referierte Forschungsposition einer restringierten futur du passé-Opposition soll nun einer eingehenderen Prüfung unterzogen werden, wobei auch auf den Basiswert der Perspektivgebundenheit zurückzukommen ist. Um das Regelwerk und die Ausnahme des „conditionnel historique“ näher zu hinterfragen, müssen zunächst die postulierten Restriktionen des futur simple du passé in der Erzählerrede genauer untersucht werden. Wenn das futur simple du passé in Strukturen, die keine Protagonistenrede darstellen, grundsätzlich nicht möglich sein sollte, dann hätte diese Restriktion eine andere Qualität als die bisher in den Beispielen beschriebenen Einschränkungen. Denn im style indirect (libre) sind beide futur du passé-Formen prinzipiell möglich, der Kontext kann jedoch eine Präferenz für eine Form festlegen bzw. eine Form völlig ausschließen. Geht z. B. aus dem Kontext hervor, daß die nachzeitige Handlung bereits aktuell konditioniert ist, dann scheidet das futur simple du passé aufgrund seiner Virtualität aus. Im Falle der Erzählerrede aber wäre die Restriktion nicht durch die Semantik der Konditionierung ausgelöst, sondern struktural bedingt: das futur simple du passé wäre auf Äußerungen eines Protagonisten in der Vergangenheit festgelegt und stünde bei Erzählerrede, unabhängig von der Art der Konditionierung, generell nicht zur Verfügung.

Um diese Restriktion zu überprüfen, wurden Beispiele in einer Testreihe Umformungen und Sprecherbefragungen unterzogen. Dabei wurde zunächst getestet, ob das in der Erzählerrede verwendete futur périphrastique du passé nicht doch durch ein futur simple du passé kommutiert werden kann. In einem zweiten Schritt wurde dann durch syntaktische Unterordnung unter ein verbum dicendi die Erzählerrede in indirekt wiedergegebene Protagonistenrede umgewandelt. Durch diese Varianten wird dann nachprüfbar, inwiefern mögliche Restriktionen für die Formen in Erzählerrede und Protagonistenrede parallel funktionieren. Für diese Testreihe führe ich zwei repräsentative Beispiele und ihre Varianten an:

(20) L'hiver s'acheva. Les premières restrictions apparurent. Bientôt, on (allait nous distribuer - nous distribuerait) les cartes de pain (Beauvoir 1960, S. 497).

37 Flydal 1943, 65; Nilsson-Ehle 1944, 87; Sten 1952, 70 f.; Togeby 1982, 382, 402.

38 Nilsson-Ehle 1944, 87; Togeby 1982, 386; vgl. auch Damourette/Pichon 1936, 429430 (Beispiele).

39 Imbs 1960, 66; Togeby 1982, 382, 402; Nilsson-Ehle 1944, 55-57, 62-64, 77, 86-88.

40 Nilsson-Ehle 1944, 62-64, $86 \mathrm{f}$. 
(21) L'hiver s'acheva. Les premières restrictions apparurent. L'administration nous informa que, bientôt, on (allait nous distribuer - nous distribuerait) les cartes de pain.

(22) Il (allait encore pleuvoir - *pleuvrait encore), le jour était d'une blancheur livide qui aveuglait. ${ }^{41}$

(23) On l'avertit qu'il (allait encore pleuvoir - *pleuvrait encore), le jour était d'une blancheur livide qui aveuglait.

Im für zahlreiche Beispiele repräsentativen Textausschnitt (20) wird anders als erwartet das futur simple du passé vollkommen akzeptiert und von befragten Sprechern in keiner Weise als markiert empfunden. In (22) wird das futur simple $d u$ passé abgelehnt, allerdings mit dem Hinweis auf die aktuelle Konditioniertheit des Regens. Diese Parallele zur Protagonistenrede wird durch die Umformungen bestätigt. So ergeben sich in (20) und (21) bzw. (22) und (23) durch die futur $d u$ passé-Formen unabhängig von den Strukturen der Erzählerrede oder Protagonistenrede die gleichen Nuancen. In (20) und (21) erscheint das Verteilen der Brotmarken im futur périphrastique du passé bereits aktuell konditioniert, im futur simple du passé dagegen hypothetischer. In (22) und (23) schließlich wirken für das futur simple du passé die gleichen Kontextfaktoren restringierend, nämlich der grelle Himmel als Vorstufe des Regens. Die Restriktion des futur simple $d u$ passé in (22) ist damit nicht übergeordneter strukturaler Natur, sondern erklärt sich wie in der zuvor dargelegten Futuropposition durch die Konditionierung.

In Absetzung vom dargelegten Forschungskonsens belegen Beispielvarianten und Sprecherbefragungen also, daß die von futur périphrastique du passé und futur simple du passé geleisteten semantisch-pragmatischen Nuancierungen auch in der Erzählerrede gegeben sind, in der die Forschung das futur simple du passé bisher struktural ausgeschlossen hat ${ }^{42}$. Damit sind beide futur du passé-Formen in Kontexten der Protagonistenrede und der Erzählerrede möglich - zu präzisieren bleibt noch, wie die Opposition von futur simple du passé und futur périphrastique du passé dann im letztgenannten Fall ausgestaltet ist. Den Gründen für die konstatierte Fehleinschätzung durch die Forschung könnte nur eine diachronisch angelegte Sichtung von Beispielmaterial nachgehen. Neben der Möglichkeit, daß diese Verwendung des futur simple du passé schlicht übersehen wurde, erscheint es auch denkbar, daß es sich um eine jüngere Entwicklung handelt, die noch nicht Eingang in die Tempusforschung gefunden hat. Diese Diskrepanz mit der Forschung wirft natürlich auch die Frage auf, wie lange das futur simple du passé in Kontexten der Erzählerrede schon ein droit de cité innehat und über welche

41 Damourette/Pichon 1936, $324 \mathrm{f}$.

42 Diese Verwendung in der Erzählerrede ist ein Grund mehr, das futur simple du passé - ebenso wie das futur périphrastique du passé oder andere Tempusformen - nicht als Charakteristikum für den style indirect libre einzustufen: darauf verweisen auch Bally 1912, 556, 597; Bally 1914, 456 f.; Stempel 1972, 322; Lebsanft 1981, 67. Die Gegenposition vertritt G. Steinberg 1971, 83, 199, der von der Beschränkung des futur simple du passé auf Protagonistenrede ausgeht und daraus zirkelhaft schließt, daß die Form dann den style indirect libre ,in hohem Maße“ charakterisiert. 
Textsorten die Verbreitung verlief ${ }^{43}$. Eine solche Analyse der Einbürgerung des futur simple du passé berührt dann auch den Komplex von Mündlichkeit und Schriftlichkeit, scheint doch in mündlichen Erzählungen das futur simple du passé kaum Verwendung zu finden - das Kontinuum von Nähe- und Distanzsprache wäre in der Fragestellung also mit zu berücksichtigen ${ }^{44}$.

Dieser Ausgriff auf die Erzählerrede erweitert auch das Konzept des Perspektivträgers, das in style indirect und style indirect libre auf den Protagonisten fixiert war $^{45}$. Die Funktion des Perspektivträgers kann nun nicht nur von einem Protagonisten, sondern auch von einem Erzähler erfüllt werden, der aus der Referenzsituation in der Vergangenheit heraus Nachzeitiges evoziert. Ein früher, dann jedoch in der Forschung nicht mehr beachteter Hinweis auf den Sprecher bzw. Erzähler als Träger der Perspektive findet sich bei Tobler, der bei Beispielanalysen dem futur du passé einen Blick in die Zukunft zuschreibt, der von „Personen der Vergangenheit", aber auch vom Sprecher vollzogen werden kann ${ }^{46}$ - eine Präzisierung, die in Forschung und Grammatiken offensichtlich nicht weiter rezipiert wurde. Konstitutiv für die perspektivisch gebundenen futur simple du passé und futur périphrastique du passé ist damit die Perspektive eines Sprechers in der Vergangenheit, der sich zu für ihn zukünftigen Sachverhalten äußert - die Rolle des Perspektivträgers kann aber mit dem Protagonisten oder dem Erzähler besetzt sein.

Im folgenden soll durch Beispielanalysen und Sprecherbefragungen überprüft werden, wie die Opposition von futur simple du passé und futur périphrastique du passé in Kontexten der Erzählerrede gestaltet ist und inwiefern sich Modifizierungen zu den in Abschnitt 3 analysierten Strukturen der Protagonistenrede ergeben. Die Interpretation der Textausschnitte zeigt, daß auch in der Erzählerrede, ob in historischen oder in anderen Textsorten, das Prinzip der Konditionierung die Tempusverwendung bestimmt:

(24) Cependant mon grand-père André ne songea pas une seconde à refuser une aussi glorieuse mission. Il embrassa donc sa chère femme, puis ses quatre enfants, bénit par avance le cinquième qui (allait naître - *naîtrait - naîtrait bientôt - allait naître bientôt) et monta vers la gare, [...] (Pagnol 1988c, S. 30).

Da die Ehefrau bereits schwanger ist, kann hier nur das teilaktuelle futur périphrastique du passé verwendet werden - das futur simple du passé würde ja aussagen, daß das Kind irgendwann einmal geboren wird. Ergänzt man nun das futur simple du passé durch das Adverb bientôt, dann ist die Form akzeptabel, da

43 In der Forschung finden sich unterschiedliche Bewertungen des futur simple du passé außerhalb der Protagonistenrede. Während Tobler ${ }^{2} 1906,146$ das futur simple du passé in ähnlichen Beispielen bereits bei ,gebildeten Franzosen“ antrifft, stuft Martin 1971, 125 diesen Typ doch etliche Jahre später noch als stark markiert ("choquant") ein.

44 Koch/Oesterreicher 1990, 8-12. Vgl. auch Schrott 1997, 138-141.

45 Vgl. Flydal 1943, 65, 67, der dem futur simple du passé eine Protagonistensicht zuschreibt.

46 Tobler ${ }^{2} 1906,141$; Beispiele $143 \mathrm{f}$. 
das Adverb den nachzeitigen Sachverhalt orientiert und die Nicht-Orientiertheit der Tempusform so kompensiert. Diese Nuance der Teilaktualität belegt auch der nächste Ausschnitt aus einem historischen Text:

(25) Mais on avait appris dans l'Europe frémissante que l'empereur n'était plus invincible. Napoléon (allait essayer - ?essayerait) de lui prouver le contraire en se rendant lui-même en Espagne pour redresser la situation [...] (Villat 1947, S. 133 f.).

Da die Aufstände in Spanien als aktuelle conditio in der vergangenen Referenzsituation schon existent sind, ist der Versuch der Disziplinierung teilaktuell und nachzeitig zur Referenzsituation orientiert: Napoléon wird den Versuch zu einem bestimmten Zeitpunkt unternehmen und nicht irgendwann später.

Im folgenden Beispiel dagegen wird im Original das futur simple du passé verwendet:

(26) Bien qu'il fît mine de traiter les femmes à l'emporte-pièce il leur attribuait, sans qu'il se l'avouât, quelque sorcellerie. [...]. Quand, après le divorce, il (se rendrait - *allait se rendre) chez Joséphine, [...], il (la préviendrait - *allait la prévenir) de sa visite afin de ne pas se prendre les pieds dans quelques amoureux (Audiberti 1961, S. 184).

Da in der Referenzsituation die Scheidung noch nicht erfolgt ist, sind die Besuche Napoléons bei der späteren Gemahlin Joséphine noch virtuell konditioniert. Das futur simple du passé leistet damit einen Blick auf Ereignisse nachzeitig zu einem anderen Sachverhalt und enthält damit auch die Ereignisstruktur der Ulteriorität, so daß eine Kommutation durch das futur périphrastique du passé nicht möglich ist.

Diese Ereignisstrukturen der Kontiguität und Ulteriorität modellieren sich auch im folgenden Textbeispiel heraus:

(27) Patrick Demouy nous contera aussi la longue histoire de ce sanctuaire, de la modeste chapelle Saint-Christophe magnifiée par la sépulture de cet homme d'exception que fut Saint Remi, mort en 533. Cette sépulture (allait être serait) en effet à l'origine d'une histoire tourmentée [...] (Amopa, S. 39).

Das futur périphrastique du passé des Originals versprachlicht, daß die bewegte Geschichte in der Bedeutung dieser Grabstätte gründet und unmittelbar nach deren Errichtung als prozeßhafte Entwicklung beginnt. Das futur simple du passé dagegen würde ausdrücken, daß die „histoire tourmentée“ erst nachzeitig zu einem anderen Sachverhalt einsetzt, etwa nach einer Phase, in der die Grabstätte zunächst einmal in Vergessenheit gerät.

Im Verlauf der Beispielanalysen und Sprecherbefragungen zeichnete sich $a b$, daß auf der Basis der aktuellen und virtuellen Konditioniertheit der nachzeitigen Sachverhalte im Kontext der Erzählerrede weitere Nuancen entstehen, die die Perspektive des Erzählens noch weiter ausgestalten:

(28) Bref, ce nouveau départ dans la vie fut préparé avec autant de soin que le placement d'un spoutnik sur son orbite, et (j'allais bientôt découvrir - je découvrirais bientôt) que j'entrais, en effet, dans un autre univers (Pagnol 1988c, S. 195). 
Da der Übertritt auf das lycée schon aktuell konditioniert ist und auch zu einem konkreten Zeitpunkt erfolgt, wird im Original das futur périphrastique du passé verwendet. Das an sich nicht orientierte futur simple du passé ist jedoch auch akzeptabel, da die Orientiertheit durch das Adverb bientôt gesichert ist. In diesem Textausschnitt ergibt sich nun auf der Basis der Semantik der Konditionierung aber noch eine weitere Differenzierung. Nach Aussagen befragter Muttersprachler wird beim futur simple du passé ein mehrwissender Erzähler evoziert, der über die Referenzsituation hinaus blickt.

Diese Nuance bildet sich im nächsten Textausschnitt noch prägnanter aus, in dem es um die Ehe Napoléons mit Marie-Louise von Habsburg geht:

(29) De la débutante il s'institua le maître d'école sexuel [...]. L'avenir (confirmerait - ?allait confirmer) que la molle nièce de Charles-Quint, toute de mièvrerie nébuleuse, n'était pas du métal requis pour une active vertu (Audiberti 1961, S. 186 f.).

Erzählt wird der Beginn der Ehe Napoléons mit einem Ausblick auf das spätere Verhalten der charakterschwachen Ehefrau. Das futur simple du passé betont hier die in der Referenzsituation noch virtuelle Natur der Vorhersage: zum Zeitpunkt der Heirat deutete noch keine aktuelle conditio auf das spätere Verhalten hin. Die künftige Entwicklung kann damit nur ein Sprecher bzw. Erzähler wahrnehmen, der über den Bereich der aktuell konditionierten Sachverhalte hinweg in die $\mathrm{Zu}$ kunft blickt.

Diese Perspektivierung ist nun keinesfalls auf historische Texte wie (29) beschränkt, sondern findet sich geläufig in erzählenden Texten:

(30) En épousant Paul, Nelly pensait que la jalousie pimentait l'amour. Le jour où Paul lui fait sa première scène, elle s'est sentie flattée: „Mais tu es jaloux!“, lui a-t-elle dit en éclatant de rire, „cela prouve que tu tiens à moi.“ L'innocente était loin de se douter que l'attention exclusive que lui portait son compagnon (allait l'enfermer - l'enfermerait) dans un huis clos terrifiant (Nouvel Obs. 1994, S. 4).

Im futur périphrastique du passé erscheint das fatale Ende der Entwicklung als aktuell im Verhalten des Ehemannes konditioniert, das futur simple du passé dagegen versprachlicht die Isolation als ein Ergebnis, das irgendwann später eintrat und in der Referenzsituation noch nicht als Vorstufe gegeben war.

Diese Differenzierung der Erzählhaltung illustriert auch der nächste Textausschnitt, in dem es um die Behandlung eines Verletzten geht:

(31) Et Félix Lacail prit le chemin de l'hôpital sous l'inculpation, non de meurtre, mais de démence, formulée par un médicin à doctrines, qui destinait les gens au cabanon à deux heures du matin, au bord d'un talus et à la lueur d'une lampe de poche. Un autre médicin (allait intervenir - interviendrait), le docteur Lavigerie, qui était un ami assez intime des Euffe de Sassenage, par la haute protection de Claire, femme à s'enticher (Chevallier 1968, S. 242 f.).

Das futur périphrastique du passé versprachlicht das Eingreifen des anderen Arztes als teilaktuell, während das futur simple das Hinzuziehen von Lavigerie als virtuelle Handlung, irgendwann nachzeitig zur Referenzsituation, versprachlicht. 
Was die Erzählhaltung betrifft, wird das futur périphrastique du passé mit einem Sprecher verknüpft, der aus der Referenzsituation heraus auf die weitere Entwicklung schließt und damit ,innerhalb der Erzählung' steht. Das futur simple du passé mit seinem Ausgriff auf eine virtuelle Zukunft dagegen läßt auf einen Perspektivträger in der Vergangenheit schließen, der auch virtuelle nachzeitige Sachverhalte kennt und damit auch über Wissen jenseits der Referenzsituation verfügt. Ein solches Mehrwissen hat dann Affinität zu einer ,allwissenden' Erzählerfigur, die zusammen mit dem Leser in die Zukunft blickt und über diese Referenzsituation hinaus kommentierend Ausblicke gewähren kann.

Im folgenden soll noch präzisiert werden, wie der Ausdruck einer Erzählhaltung mit der Semantik der futur du passé-Formen verknüpft ist. Bei meinen Überlegungen gehe ich zuerst von einem Beispiel aus, in dem nur das futur périphrastique du passé möglich ist, und von seiner Variante, die beide Formen zuläßt:

(32) Dès avant minuit, la situation parut tout à fait critique. La lutte (allait devenir - *deviendrait) impossible. Trois crises, d'une extrême violence, venaient d'avoir lieu, coup sur coup, lorsque'une quatrième se déclara (Martin du Gard 1972a, S. 309).

(33) Vers midi, la situation parut s'améliorer. Cependant, une nouvelle crise (allait se déclarer - se déclarerait) dans la nuit.

Da in (32) durch die „situation critique“ bereits aktuell konditioniert ist, daß der Kampf um das Leben des Kranken unmöglich wird, scheidet das futur simple $d u$ passé hier aus. In (33) dagegen läßt der Kontext sowohl aktuelle als auch virtuelle Konditionierung zu. In der Variante ergibt sich dann auch kontrastiv der erwartete Unterschied hinsichtlich der Erzählhaltung. Beim futur périphrastique du passé schließt der Perspektivträger aus der erzählten Referenzsituation heraus auf die nachzeitige Entwicklung, wodurch der Eindruck eines Erzählens aus dieser Situation heraus entsteht. Beim futur simple du passé dagegen greift der Erzähler in den Bereich virtueller Sachverhalte aus, die aus der Referenzsituation heraus nicht inferiert werden können - ein Ausblick, der in geeigneten Kontexten mit einer mehrwissenden Erzählerfigur verknüpft wird. Für den Zusammenhang von Erzählhaltung und Tempussemantik ist nun entscheidend, daß die beschriebenen Effekte der Erzählhaltung auf der Basissemantik der Konditionierung aufbauen. Im Fall des futur périphrastique du passé fokussiert die aktuelle conditio die Referenzsituation, in der der ,Sprecher in der Vergangenheit" sich äußert, und erzeugt so die Nuance eines Erzählens aus der Referenzsituation heraus. Das virtuelle futur simple du passé dagegen kann einen Erzähler suggerieren, der über die Referenzsituation und die in ihr angelegten Sachverhalte hinaus nachzeitige Entwicklungen aufspüren kann. Diese Ableitung der Erzählhaltung aus der Semantik der Konditionierung wird auch von der Tatsache untermauert, daß in (32) nicht das futur simple du passé eingesetzt werden kann, um die geschilderte Nuance eines mehrwissenden Erzählers zu erzeugen. Ausschlaggebend für die Tempusverwendung ist nämlich die aktuelle oder virtuelle Konditionierung als Basiswert der Formen und nicht die als Kontexteffekt einzustufenden Differenzierungen der Erzählperspektive. 
In Texten, wo sich der Kontexteffekt des mehrwissenden Erzählers ergibt, steht das futur simple du passé in Konkurrenz zu anderen Formen, die ebenfalls die Perspektive eines mehrwissenden Erzählers versprachlichen: das futur historique und die devoir-Periphrase. Diese Ausdrucksmöglichkeiten sollen in einem Exkurs zumindest kurz vorgestellt werden. Das folgende Beispiel ist repräsentativ für das semantisch-pragmatische Profil der in dieser Funktion im imparfait verwendeten devoir-Periphrase ${ }^{47}$ :

(34) Le mulet fut remis entre les brancards, et nous sortîmes du village: alors commença la féerie et je sentis naître un amour qui (devait durer - durerait allait durer) toute ma vie (Pagnol 1988b, S. 87).

Die devoir-Periphrase des Originals suggeriert einen Blick in die Zukunft bis ans Ende des Lebens und assoziiert einen Erzähler, der das Ende der Geschichte kennt und in der Retrospektive wei $\beta$, daß alles so und nicht anders kommen mußte. Das futur simple du passé leistet durch das Wissen um eine virtuelle Zukunft die gleiche Nuance, allerdings schwächer ausgeprägt. Das futur périphrastique du passé dagegen verändert die Perspektive deutlich, indem es das Erwachen der Liebe und die Prospektivität dieses Prozesses hervorhebt.

Die Verwendung des futur historique kann hier nur skizziert werden ${ }^{48}$. Beim futur historique, das von futur simple und futur périphrastique geleistet werden kann, versetzt sich der Erzähler in die Vergangenheit und läßt dabei die vergangene Referenzsituation als fingiertes ego-hic-nunc erscheinen, von dem aus nachzeitige Sachverhalte dann durch die Futura versprachlicht werden ${ }^{49}$.

(35) Dès cette époque, il vénère également Racine, Molière et La Fontaine. De cette culture classique il (gardera - va garder) une empreinte ineffaçable.

Durch das einleitende historische Präsens und die Zeitangabe dès cette époque wird in der Vergangenheit eine Referenzsituation etabliert, zu der das futur simple dann Nachzeitigkeit versprachlicht. Durch die Verwendung von présent und futur simple wird das ego-hic-nunc des Sprechers in die Vergangenheit verschoben und der Erzähler blickt von dieser fingierten Sprechsituation aus in die Zukunft. Im Unterschied $\mathrm{zu}$ den futur du passé-Formen versprachlicht das futur historique bereits vergangene Sachverhalte nicht nur als nachzeitig zur Referenzsituation, sondern präsentiert diese als zukünftig und erzeugt so die Fiktion einer Versetzung in die Vergangenheit. Das futur historique wird nun auch geläufig mit den futur du passé-Formen kombiniert, wenn in erzählenden Texten Nachzeitiges thematisiert wird:

(36) Jérôme [...] est peut-être celui en qui les goûts du parvenu se marquent avec le plus d'évidence, [...]. Il aurait peut-être, moins encore que les autres, le sens de l'Etat. [...]. Mais, comme la justice est une vertu morale que la vie ne

47 Vgl. hierzu auch Sten 1952, 75; Nilsson-Ehle 1944, 66-75, 81-82; Flydal 1943, 65; Sten 1952, 75-77; Imbs 1960, 69-70; Togeby 1982, 402, $404 \mathrm{f}$.

48 Tobler ${ }^{2} 1906$, 136-140; Flydal 1943, 59, 65; Imbs 1960, 46f., 68; Togeby 1982, 386.

49 Beispiel Togeby 1982, 386. Vgl. zu diesem Typ auch Flydal 1943, 65-67; Sten 1952, 62; Imbs 1960, 46 ff.; Togeby 1982, 386. 
connaît pas, c'est justement Jérôme qui aura pour épouse la plus noble et la plus fidèle. La grassouillette Catherine de Wurtemberg allait si bien s'attacher à son époux, à l'Empereur et à la France, qu'elle a préféré tout perdre à l'abandon de ses devoirs d'épouse. Catherine, née princesse, s'est trouvée la plus croyable, quand elle a un jour déclaré que, si elle venait à perdre Jérôme, pourtant découronné, c'est sur le rocher de Sainte-Hélène qu'elle choisirait d'aller mourir (Beer 1961, S. 143 f.).

Die Biographie Jérômes beginnt mit einem im futur simple du passé (,,il aurait“) versprachlichten Ausblick auf seine mangelnden staatsmännischen Fähigkeiten es geht hier um seine Zeit als König von Westfalen. Der futur du passé-Form folgt dann ein Ausblick auf die Eheschließung, der durch das futur simple historique (,qui aura") geleistet wird. Nach dem eingeschobenen futur historique verwendet der Erzähler erneut ein futur du passé. Das prospektive futur périphrastique du passé drückt hier die allmählich sich entwickelnde Liebe Catherines zu ihrem nichtsnutzigen Ehemann aus. Der Textausschnitt schließt mit einem futur simple du passé, das allerdings keine Erzählerrede mehr darstellt, sondern die Worte Catherines wiedergibt: Nach dem noch virtuellen Tod Jérômes wird sie ihr Leben im freigewählten Exil beschließen. Die Juxtaposition von Erzählerrede und Protagonistenrede illustriert dabei noch einmal die identischen Gebrauchsbedingungen in beiden Kontexttypen.

Der Textabschnitt dokumentiert so abschließend auf engem Raum, daß der Blick eines Perspektivträgers aus der Vergangenheit in die Zukunft durch eine Vielfalt sprachlicher Mittel geleistet werden kann. Nachdem die futur du passéFormen als perspektivisch gebundene Formen analysiert wurden, ist nun noch auf einen markierten Verwendungstyp des futur périphrastique du passé einzugehen.

\section{Prospektiver Aspekt - ein Verwendungstyp des futur périphrastique du passé}

Bei der kontrastiven Behandlung von futur simple du passé und futur périphrastique du passé in der Protagonistenrede zeigte sich bereits, daß die beim futur périphrastique gegebene aspektuelle Komponente der Prospektivität durch den Kontext akzentuiert werden kann. Eine solche Aspektbetonung findet sich nun auch bei einer großen Zahl von futur périphrastique du passé Verwendungen in Kontexten der Erzählerrede. Die Beispiele entsprechen meist dem Muster der folgenden Ereignisstruktur:

(37) A petits pas prudents, Elisabeth s'avança vers l'escalier. Elle (allait mettre *mettrait) son pied sur la première marche, quand la voix d'Amélie arrêta son mouvement: [...] (Troyat 1956, S. 53).

Elisabeth ist gerade im Begriff, den Fuß auf die erste Treppenstufe zu setzen, wird aber von Amélie davon abgehalten. Der durch das futur périphrastique du passé versprachlichte Sachverhalt ist also auf dem Wege der Zieleinlösung, als ein weiteres Geschehen eintritt und den imminenten Sachverhalt verhindert. Diese Nuance eines Sachverhalts, der als Vorstadium gerade in die Phase der 
Realisierung eintritt, kann ausschließlich vom futur périphrastique du passé geleistet werden.

Die dem Textausschnitt zugrundeliegende Ereignisstruktur entspricht damit dem Inzidenzschema, wie es Pollak bei den Vergangenheitstempora beschreibt ${ }^{50}$ : ein im imparfait versprachlichter Sachverhalt war im Gange, als ein anderer, durch eine perfektive Form (passé simple, passé composé) ausgedrückter Sachverhalt eintrat. Überträgt man das Inzidenzmuster auf die Verwendungen mit dem futur périphrastique du passé, dann ergibt sich die Variante, daß ein Sachverhalt im futur périphrastique du passé als Vorstadium teilaktuell war, als ein anderer Sachverhalt eintrat. Wie das imparfait, so beinhaltet auch das futur périphrastique $d u$ passé in seiner Prospektivität einen imperfektiven Wert und kann damit die Nuance eines schon und noch gegebenen Zustandes leisten. Zu unterstreichen ist jedoch, daß beide Typen von Imperfektivität semantisch differenziert sind:

(38) Jacques (ouvrait - allait ouvrir) la bouche pour crier: „Moi si!““ lorsqu'il aperçut, debout au milieu de la pièce, son frère, qui s'était retourné et qui le considérait fixement (Martin du Gard 1972b, S. 203).

Während das imparfait impliziert, daß Jacques schon mit halboffenem Mund dasitzt, hat beim futur périphrastique du passé die Handlung des Öffnens noch nicht begonnen, Jacques ist aber drauf und dran den Mund zu öffnen, als er den Bruder bemerkt ${ }^{51}$.

Diese Aspektfokussierung und das Inzidenzmuster finden sich häufig in Temporalsatzgefügen ${ }^{52}$, sind aber nicht an diese Struktur gebunden, wie das folgende Beispiel belegt:

(39) Paul (allait recommencer - *recommencerait) la chasse aux larmeuses, mais ma mère l'en dissuada, par quelques paroles pathétiques qui lui mirent les larmes aux yeux (Pagnol 1988b, S. 78).

Paul will gerade die Jagd von neuem beginnen, als die Ermahnungen der Mutter ihn davon abhalten.

In den analysierten Beispielen ist die starke Fokussierung der Prospektivität durch den Kontext bedingt und gewinnt durch das Inzidenzschema zusätzliche Prägnanz. Darüber hinaus könnte die Hervorhebung der prospektiv-imperfektiven Aspektkomponente beim futur périphrastique du passé auch durch das System der Vergangenheitstempora bedingt sein, in dem die imperfektiv-prospektive Komponente kontrastiv durch perfektiv markierte Formen wie passé composé bzw. passé simple hervorgehoben werden dürfte.

Neben der starken Hervorhebung der aspektuellen Komponente fällt auf, daß bei den meisten Beispielen dieses Typs die für beide futur du passé-Formen konstitutive perspektivische Gebundenheit als Blick eines Perspektivträgers nur sehr schwach ausgeprägt ist ${ }^{53}$. Dies zeigt sich am signifikantesten bei den Temporalsatzgefügen:

50 Pollak $1960,129-133$, bes. $131 \mathrm{f}$.

51 Vgl. Flydal 1943, 17, 20; Sten 1942, 241; Martin 1971, 139.

52 Vgl. Togeby 1982, 318-332, 360.

53 Eine ähnliche Beobachtung findet sich bei N. M. Steinberg 1972, 177-178. 
(40) Elle touma le commutateur. Une lumière vive l'éblouit. Elle (allait s'avancer - *s'avancerait) vers l'armoire, quand son cœur se crispa et ses jambes fléchirent (Troyat 1956, S. 387).

Das Mädchen will auf den Schrank zugehen, als es plötzlich erschrickt und stehenbleibt. Im Vordergrund steht das in der Referenzsituation gegebene Vorstadium, dem gegenüber die temporalen Komponenten der Nachzeitigkeit und der perspektivischen Gebundenheit zurücktreten.

Dieses Phänomen ist nun nicht auf den Fall des Temporalsatzgefüges beschränkt, sondern ist immer dann zu beobachten, wenn die Aspektkomponente hervorgehoben wird:

(41) „Hé! l'ami!“ Je vis un garçon de mon âge, qui me regardait sévèrement. „Il ne faut pas toucher les pièges des autres, dit-il. Un piège, c'est sacré!“ - „Je (n'allais pas le prendre - *ne le prendrais pas), dis-je. Je voulais voir l'oiseau“ (Pagnol 1988a, S. 12).

Der Sprecher bezieht sich hier auf sein Verhalten wenige Momente zuvor: als Lili ihn beobachtete, bestand in dieser Referenzsituation nicht die Intention, den Vogel zu stehlen. Das futur périphrastique du passé versprachlicht auch hier eine Vorstufe, ohne eine deutliche Perspektive der Nachzeitigkeit zu etablieren.

Diese weitgehende Reduzierung des futur périphrastique du passé auf den prospektiven Aspekt eines Vorstadiums nähert diesen Verwendungstyp nun einem semantischen Konzept an, das als proximative beschrieben wird. Heine ${ }^{54}$ faßt den proximative als nicht-deiktische Aspekt-Kategorie auf, die Sachverhalte versprachlicht, die nahe an der Realisierung sind, deren Realisierung aber noch nicht begonnen hat ${ }^{55}$. Dem futur périphrastique ist nun durch die aktuelle conditio eine prospektive Aspektkomponente inhärent, die bei entsprechender Akzentuierung durch den Kontext eine dem proximative verwandte Nuance erzeugt. In diesen Verwendungen kommt dann der prospektiv-imperfektive Aspekt zum Tragen, aus dem sich die Futura diachronisch entwickelt haben ${ }^{56}$. Da der prospektive Aspekt auf diese Weise mit der Basisbedeutung des futur périphrastique du passé untrennbar verknüpft ist, kann im Französischen keine eigene Kategorie des proximative angesetzt werden. Allerdings kann sich das futur périphrastique du passé bei extremer Fokussierung des Aspekts und Ausblendung der Temporalität dem Konzept des proximative stark annähern - hier könnte der proximative als kontextbedingter Verwendungstyp des futur périphrastique du passé betrachtet wer$\operatorname{den}^{57}$.

54 Heine 1994, 36: „In more general terms, the function concerned may be said to define a temporal phase located close to the initial boundary of the situation [...]". Zur Entwicklung des proximative vgl. Heine 1994, 37-40, 42, 44 und Romaine 1997, 15-17.

55 Der proximative ist damit als Ausdruck noch nicht realisierter Sachverhalte der Futurität konzeptuell verwandt, impliziert aber anders als futurische Tempora keine deiktische Situierung. Vgl. Heine 1994, 42-44; vgl. auch Romaine 1997, 40-42, 45, 62.

56 Flydal 1943, 26, 30, 61, 107; Fleischman 1982, 85, 99.

$57 \mathrm{Zu}$ den möglichen Verbindungen zwischen Futur, Aspekt und proximative vgl. Heine 1994, 42-44; Romaine 1997, 40-42, 45, 62. 
In den gesichteten Texten überwiegen nun Beispiele, in denen die Fokussierung der Vorstufe mit der kontextuellen Inferenz verbunden ist, daß die Realisierung des bereits teilaktuellen Sachverhaltes verhindert wird. Diese Besonderheit kann durch die dargelegte Aspektfokussierung erklärt werden. Die Betonung der Anfangsphase legt nämlich die Inferenz nahe, daß der Sachverhalt selbst geringere Bedeutung hat, was häufig dann der Fall ist, wenn der Sachverhalt über das akzentuierte Vorstadium nicht hinaus kam und daher in seiner Gesamtheit gar nicht im Zentrum stehen kann. Auf dieser Basis entsteht dann die kontextuelle Implikatur eines im Vorstadium abgebrochenen Sachverhalts, die aber nicht zur Semantik des futur périphrastique du passé gehört, wie die nächsten Beispiele belegen:

(42) Sur le trottoir, où Jacques aborda, un couple (allait se séparer - *se séparerait). L'homme et la femme se regardaient une dernière fois. [...] Alors, [...] il recula, sans la quitter des yeux; puis, se retournant soudain, il s'élança vers la gare. Et elle, au lieu de le rappeler, au lieu de le suivre du regard, elle fit un brusque demi-tour, et se sauva (Martin du Gard 1972b, S. 320).

(43) Le procès-verbal, pour les gens de mon village, c'était le déshonneur et la ruine. Un gendarme d'Aubagne avait été tué dans la colline, par un brave homme de paysan, parce qu'il (allait lui faire - *lui ferait) un procès-verbal (Pagnol 1988a, S. 195).

In (42) ist durch die Situation der Abschied aktuell konditioniert und der Moment vor der Trennung wird durch die Prospektivität akzentuiert. In der Folge trennen sich die Eheleute dann auch unmittelbar nach dieser dramatischen Situation. Im Fall (43) des bedauernswerten Gendarmen von Aubagne dagegen kommt das Schriftstück über das Vorstadium nicht hinaus, da der Bauer die geschilderte drastische Gegenmaßnahme ergreift.

Aus den Beispielanalysen folgt, daß die Ereignisstruktur einer im Vorstadium abgebrochenen Handlung das Ergebnis einer Interaktion der aspektuellen Markierung des futur périphrastique du passé mit dem Kontext ist und daher nicht unter die Bedeutung des futur périphrastique du passé subsumiert werden darf ${ }^{58}$. Diese Ereignisstruktur hat vielmehr den Status einer konversationellen Implikatur, die durch Kontextveränderung auch wieder außer Kraft gesetzt werden kann ${ }^{59}$. Nicht auszuschließen ist allerdings, daß die konversationelle Implikatur bei diesen Verwendungen zunehmend mit dem futur périphrastique du passé verknüpft wird und möglicherweise in einem späteren Stadium einmal so fest mit der Form assoziiert wird, daß die konversationelle Implikatur konventionalisiert und Teil der Tempusbedeutung wird. In der Grammatikalisierungsforschung wird dieser metonymische Mechanismus, der auf einer Kontiguitätsrelation fußt, mehrmals beschrieben ${ }^{60}$. Tritt eine Form häufig in Kontexten auf, die eine bestimmte Inferenz beinhalten, dann besteht eine Kontiguität zwischen Form und Inferenz, die be-

58 Vgl. auch Fleischman 1982, 88 und 180 n. 52.

59 Vgl. dazu auch Comrie 1985, $18 \mathrm{f}$., 24.

60 Traugott/König 1991, 210-212; Traugott/Hopper 1993, 72-74, 75f., 80-83; Heine/ Claudi/Hünnemeyer 1991, 164-167. 
wirkt, daß beide Größen fest verknüpft werden. Die Implikatur kann dann im Proze $ß$ der ,context-induced reinterpretation"61 in die Semantik der Tempusform übergehen und als Teil der Bedeutung konventionalisiert werden. Das Funktionsprofil des futur périphrastique du passé läßt nicht darauf schließen, daß diese Integration der Inferenz bereits begonnen hat, lediglich die Assoziation dieser Inferenz mit der Form konnte durch Sprecherbefragungen bestätigt werden. Für den Fall, daß die in der Grammatikalisierungsforschung beschriebene Entwicklungslinie wirksam wird, könnte das futur périphrastique du passé einen semantischen Wert gewinnen, den auch Heine ${ }^{62}$ dem letzten Entwicklungsstadium des proximative zuweist: die Semantik eines in der Vorphase abgebrochenen Sachverhalts ${ }^{63}$.

Der Verwendungstyp des futur périphrastique du passé als prospektiver Aspekt ist an eine kontextbedingte Fokussierung der Aspektkomponente geknüpft, die mit einer Defokussierung der temporal-nachzeitigen Komponente einhergeht. Diese Akzentuierung der aspektuell-prospektiven Komponente ist beim futur périphrastique du passé noch ausgeprägter als beim ebenfalls prospektiven futur périphrastique. Denn während das futur périphrastique stets temporal-futurischen Wert behält, kann die temporale Komponente beim aspektfokussierten futur périphrastique in sehr hohem Maße ausgeblendet werden.

\section{Ausblick}

Die Erarbeitung eines semantisch-pragmatischen Profils der futur du passéFormen vor der Folie der Opposition von futur simple und futur périphrastique hat gezeigt, daß die mit dem Modell der Konditionierung faßbare Futuropposition im Kontext einer Referenzsituation in der Vergangenheit ihre Gültigkeit behält und das Prinzip der Konditionierung sich auch hier in der (Nicht-) Orientiertheit der Sachverhalte, in den Ereignisstrukturen der Ulteriorität und Kontiguität und in der aspektuellen Markierung manifestiert. Diese Opposition funktioniert nun entgegen dem Bild der bisherigen Forschung nicht nur im Kontext der Protagonistenrede, sondern auch in der Erzählerrede. Entscheidend ist hier, daß das für beide futur du passé-Formen konstitutive Konzept des Perspektivträgers in der Vergangenheit von einem Protagonisten oder von einem Erzähler erfüllt werden kann. Die Gebrauchskontexte des futur simple du passé sind also auf alle Fälle perspektivischer Gebundenheit auszuweiten - sei der Träger nun ein Protagonist oder ein Erzähler.

61 Heine/Claudi/Hünnemeyer 1991, 164-167, 171, 181.

62 Heine 1994, 39; vgl. auch Romaine 1997, 16-17.

$63 \mathrm{Vgl}$. Heine 1994, 39; Romaine 1997, 5-6. Während der proximative eine reine Aspektform ist, erreicht die letzte Stufe eine neue Qualität, denn die Bedeutung einer in der Vorstufe der Zieleinlösung abgebrochenen Situation ist auf Vergangenheitskontexte beschränkt und hat damit temporalen Wert. Diese letzte Stufe der proximative-Entwicklung wird in der Forschung auch als eigene Kategorie aufgefaßt, so bei Kuteva (im Druck, zitiert nach Romaine 1997, 5-6, 45), die diese Formen als ,action narrowly averted (ANA)“ auffaßt. 
Wie das futur simple du passé, so ist auch das futur périphrastique du passé durch das Konzept der Nachzeitigkeit als einer perspektivischen Gebundenheit charakterisiert. Eine Ausnahme stellen hier aber Kontexte der Aspektfokussierung dar, in denen das futur périphrastique du passé nur noch begrenzt die Perspektive eines futur du passé ausdrückt.

Im Verlauf der Beispielanalysen wurde ferner deutlich, daß es entscheidend ist, kontextuelle Werte als Interaktion von Form und Kontext zu begreifen. Die Notwendigkeit einer exakten Trennung der Leistungen von Form und Kontext belegt das untersuchte Schema einer im Vorstadium abgebrochenen Handlung, das nicht unter die Semantik des futur périphrastique du passé subsumiert werden darf. Ebenso illustriert die Erzählerrede das Entstehen von Kontexteffekten, denn die konstatierten Erzählhaltungen können nicht als solche den futur du passéFormen zugeschrieben werden, sondern sind Effekte der Semantik der Konditionierung. Auch wenn Tempora also keine Formen des Erzählens beinhalten, haben sie in ihrer Interaktion mit Kontextfaktoren doch durch ihre Textfunktionen spezifischen Anteil an der Ausprägung bestimmter Erzählhaltungen - ein Anteil, der durch die Einbeziehung der Tempussemantik auch präzise formuliert werden kann.

Wie bereits angedeutet, stellen die behandelten Formen futur simple du passé und futur périphrastique du passé nur einen Ausschnitt der Verbalformen dar, die ,Zukunft in der Vergangenheit' versprachlichen. Die Funktion, Nachzeitiges zu einer Referenzsituation in der Vergangenheit auszudrücken, wird auch von futur simple und futur périphrastique in der Funktion futur historique und der devoirPeriphrase geleistet. Eine weiterführende Untersuchung dieser Ausdrucksformen könnte dann nicht nur paradigmatisch semantisch-pragmatische Differenzierungen der einzelnen Formen herausarbeiten, sondern auch in syntagmatischer Sicht präzisieren, wie diese verwandten Ausdrucksmittel im Text immer dann kombiniert werden, wenn aus der Vergangenheit mehr oder weniger wissenden Auges in die Zukunft geblickt wird.

Bochum, im Oktober 1997

Abkürzungsverzeichnis der Quellentexte und Fundstellen

Alain-Fournier 1971

Amopa 1997

Audiberti 1961

Beauvoir 1960

Beer 1961

Camus 1968

Camus 1972

Chevallier 1968

Martin du Gard 1972a
Alain-Fournier, Le grand Meaulnes, Arthème Fayard, Paris 1971.

Revue de l'Amopa, September 1997.

J. Audiberti, Napoléon et l'amour, in Napoléon (Collection Génies et Réalités), Hachette, Paris 1961, S. 167-187.

S. de Beauvoir, La force de l'âge, Gallimard, Paris 1960.

$\mathrm{J}$. de Beer, La grande famille, in Napoléon (Collection Génies et Réalités), Hachette, Paris 1961, S. 141-165.

A. Camus, L'étranger (Livre de poche), Paris 1968.

A. Camus, La peste, Gallimard, Paris 1972.

G. Chevallier, Les héritiers Euffe (Livre de poche), Paris 1968.

R. Martin du Gard, Les Thibault. Vol. I: La consultation - La Sorellina - La mort du père, Gallimard, Paris 1972. 
Martin du Gard 1972b

Nouvel Obs. 1994

Pagnol 1988a

Pagnol 1988b

Pagnol 1988c

Simenon 1989

Troyat 1956

Villat 1947

Bally 1912

Bally 1914

Comrie 1976

Comrie 1985

Coseriu 1976

Damourette/Pichon 1936

Dendale 1993

Fleischman 1982

Flydal 1943

Franckel 1984

Gather 1994

Gougenheim 1929
R. Martin du Gard, Les Thibault. Vol. IV: L'été 1914 (suite), Gallimard, Paris 1972.

Le Nouvel Observateur, no 1527, 10-18 février 1994.

M. Pagnol, Le château de ma mère. Souvenirs d'enfance, Editions de Fallois, Paris 1988.

M. Pagnol, La gloire de mon père. Souvenirs d'enfance, Editions de Fallois, Paris 1988.

M. Pagnol, Le temps des secrets. Souvenirs d'enfance, Editions de Fallois, Paris 1988.

G. Simenon, Maigret a peur, in Tout Simenon, Vol. VI, Presses de la Cité, Paris 1989.

H. Troyat, Les semailles et les moissons, Vol. III: La grive, Librairie Plon, Paris 1956.

L. Villat, La Révolution et l'Empire (1789-1815), Presses universitaires de France, Paris 1947.

\section{Bibliographie}

Ch. Bally, Le 'style indirect libre) en français moderne, GRM 4, S. 549-556, 597-606.

Ch. Bally, Figures de pensée et Formes Linguistiques, GRM 6, S. 405-422, 456-470.

B. Comrie, Aspect. An Introduction to the Study of Verbal Aspect and Related Problems, Cambridge.

B. Comrie, Tense, Cambridge-London-New York.

E. Coseriu, Das romanische Verbalsystem. Herausgegeben und bearbeitet von Hansbert Bertsch, Tübingen.

J. Damourette/E. Pichon, Des mots à la pensée. Essai de Grammaire de la langue française (1911-1936). Tome cinquième, verbe (fin): Auxiliaires - Temps - Modes - Voix, Paris.

P. Dendale, Le conditionnel de l'information incertaine: marqueur modal ou marqueur évidentiel?, in: G. Hilty (Hrsg.), $X X^{e}$ Congrès International de Linguistique et Philologie Romanes. Vol. I, Tübingen, S. 165-176.

S. Fleischman, The Future in Thought and Language. Diachronic Evidence from Romance, Cambridge-London-New York.

L. Flydal, (Aller > et 〈venir de s suivis de l'infinitif comme expressions de rapports temporels, Avhandlinger det Norske Videnskaps-Akademi Oslo, S. 1-119.

J.-J. Franckel, Futur, simple' et futur ,proche;, FMo. 23, S. $65-70$.

A. Gather, Formen referierter Rede. Eine Beschreibung kognitiver, grammatischer, pragmatischer und äußerungslinguistischer Aspekte, Frankfurt/M.

G. Gougenheim, Etude sur les périphrases verbales de la langue française, Paris. 
Heine 1994

\author{
Heine/Claudi/ \\ Hünnemeyer 1991
}

Helland 1994

Imbs 1960

Klum 1961

\section{Koch/Oesterreicher 1990}

Kuteva 1997

Lebsanft 1981

Lerch 1914

Martin 1971

Morgenstern 1988

Nilsson-Ehle 1944

Pollak 1960

Reichenbach 1947

Romaine 1997

Roncador 1988

Schrott 1997

Spang-Hanssen 1983
B. Heine, On the Genesis of Aspect in African Languages: The Proximative, in: K. Moore u. a. (Hrsg.), Proceedings of the 20th Annual Meeting of the Berkeley Linguistics Society. Special Session on Historical Issues in African Linguistics, Berkeley, S. 35-46.

B. Heine/U. Claudi/F. Hünnemeyer, From Cognition to Grammar: Evidence from African Languages, in: E. C. Traugott/B. Heine (Hrsg.), Approaches to Grammaticalization. Vol. I, Amsterdam/Philadelphia, S. 149-187.

H. P. Helland, Sémantique et pragmatique temporelle. Futur simple et futur périphrastique, Oslo.

P. Imbs, L'emploi des temps verbaux en français moderne. Essai de grammaire descriptive, Paris.

A. Klum, Verbe et adverbe. Etude sur le système verbal indicatif et sur le système de certains adverbes de temps à la lumière des relations verbo-adverbiales dans la prose du français contemporain, Stockholm-Göteborg-Uppsala.

P. Koch/W. Oesterreicher, Gesprochene Sprache in der Romania: Französisch, Italienisch, Spanisch, Tübingen.

T. Kuteva, On Identifying an Evasive Gram: Action Narrowly Averted, erscheint in: Studies in Language.

F. Lebsanft, Perspektivische Rededarstellung (Erlebte Rede) in Texten des französischen und spanischen Mittelalters, ZRPh. 97, S. 65-85.

E. Lerch, Die stilistische Bedeutung des Imperfektums der Rede (,style indirect libre'), GRM 6, S. 470-489.

R. Martin, Temps et aspect. Essai sur l'emploi des temps narratifs en moyen français, Paris.

C. Morgenstern, Futur im Französischen. Wissenschaftliche Übung an der Ludwig-Maximilians-Universität München, Sommersemester 1988.

$\mathrm{H}$. Nilsson-Ehle, Le conditionnel 'futur du passé et la périphrase (devait) + infinitif, in Studia Neophilologica 16, S. $50-88$.

W. Pollak, Studien zum (Verbalaspekt) im Französischen, Wien.

H. Reichenbach, Elements of Symbolic Logic, New York-London.

S. Romaine, The Grammaticalization of the Proximative in Tok Pisin, unveröffentlichtes Manuskript.

M. v. Roncador, Zwischen direkter und indirekter Rede. Nichtwörtliche direkte Rede, erlebte Rede, logophorische Konstruktionen und Verwandtes, Tübingen.

A. Schrott, Futurität im Französischen der Gegenwart. Semantik und Pragmatik der Tempora der Zukunft, Tübingen.

E. Spang-Hanssen, La notion de verbe auxiliaire, in: $M$. Herslund u. a. (Hrsg.), Analyses grammaticales. Etudes publiées à l'occasion du $50^{e}$ anniversaire de Carl Vikner, Kopenhagen, S. 5-16. 
G. Steinberg 1971

N. M. Steinberg 1972

Stempel 1972

Sten 1952

Sundell 1991

Tobler ${ }^{2} 1906$

Togeby 1982

Traugott/König 1991

Traugott/Hopper 1993

Vet 1980

Vet 1994

Vetters 1994

Wainstein 1949

Weinrich 1982

Wilmet 1970
G. Steinberg, Erlebte Rede. Ihre Eigenart und ihre Formen in neuerer deutscher, französischer und englischer Literatur, Göppingen.

N. M. Steinberg, Grammaire française, Leningrad.

W.-D. Stempel, Perspektivische Rede in der französischen Literatur des Mittelalters, in: E. Leube/L. Schrader (Hrsg.), Interpretation und Vergleich. Festschrift für Walter Pabst, Berlin, S. $310-330$.

H. Sten, Les temps du verbe fini (indicatif) en français moderne, Kopenhagen.

L.-G. Sundell, Le temps futur en français moderne, Uppsala.

A. Tobler, Vom Gebrauche des Futurum Praeteriti, in: ders., Vermischte Beiträge zur französischen Grammatik, Vol. II, Leipzig, S. 136-159.

K. Togeby, Grammaire française. Vol. II Les Formes Personnelles $d u$ Verbe. Publiée par Magnus Berg, Ghani Merad, Ebbe Spang-Hanssen, Kopenhagen.

E. C. Traugott/E. König, The Semantics-Pragmatics of Grammaticalization Revisited, in: E. C. Traugott/B. Heine (Hrsg.), Approaches to Grammaticalization, Vol. I. Amsterdam-Philadelphia, S. 189-218.

E. C. Traugott/P. J. Hopper, Grammaticalization, Cambridge.

C. Vet, Temps, aspects et adverbes de temps en français contemporain, Genf.

C. Vet, Future Tenses and Discourse Representation, in: C. Vet/ C. Vetters (Hrsg.), Tense and Aspect in Discourse, Berlin-New York, S. 49-76.

C. Vetters, Free Indirect Speech in French, in: C. Vet/C. Vetters (Hrsg.), Tense and Aspect in Discourse, Berlin-New York, S. $179-225$.

L. Wainstein, $L$ 'expression du commandement dans le français actuel, Helsingfors.

H. Weinrich, Textgrammatik der französischen Sprache, Stuttgart.

M. Wilmet, Le système de l'indicatif en moyen français, Genf. 